\title{
Lower Limb Amyoplasia in a Filipino Female: A Case Report and a Review of the Literature
}

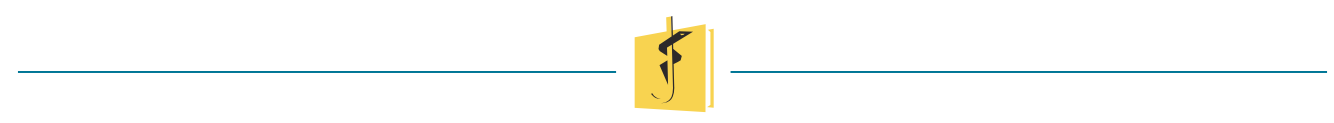

\author{
Ted Matthew P Evangelista, MD* \\ Carmelo L Braganza, $M D^{\prime}$ \\ Romel P Estillore, $M D^{2}$ \\ Jeremy James $C$. Munji, $M D^{3}$ \\ Mara Therese P. Evangelista-Huber, $M D^{4}$
}

\begin{abstract}
Amyoplasia is the most common form of arthrogryposis (multiple congenital contracture). It has an estimated incidence of one in 10,000 live births. Lower limb-amyoplasia is a specific subtype with an estimated incidence of 1 in 75,000 - 100,000 live births. There are only 85 cases of lower limb amyoplasia identified in literature, and there are no published cases from the Philippines. We discuss a rare case of lower limb amyoplasia in an 11-year-old Filipino female presenting with additional unique features of severe scoliosis (managed with spine correction surgery) and multiple skin dimples in the gluteal area. The study was performed according to the CARE guidelines for case reports.
\end{abstract}

Keywords arthrogryposis multiplex congenita, amyoplasia, lower limb amyoplasia, skin dimpling, scoliosis, case report

Ted Matthew P Evangelista

mevangelistamd@gmail.com

1,2,3 Department of Orthopedic Surgery

University of Santo Tomas Hospital, Manila, Philippines

4 Levana Dermatology Clinic, Manila, Philippines

\section{INTRODUCTION}

Arthrogryposis or arthrogryposis multiplex congenita refers to conditions with multiple congenital contractures.[1] These terms often used interchangeably, are considered a phenotypic description rather than a diagnosis. Medical literature has not been consistent in its use causing confusion in some cases.[2]

Arthrogryposis has three general categories, each with a different etiopathogenesis (ie, sporadic conditions, gene and chromosomal abnormalities, syndromes, and teratogenic pathology)[2]:

a. Amyoplasia, the classical form of arthrogryposis (the most common variant of arthrogryposis), is completely sporadic, occurring in about 1 in 10,000 live births. [3,4]

b. Distal forms of arthrogryposis are characterized by a predominant lesion in the distal segments of the limbs (hand, foot) with/without maxillofacial anomalies. All have autosomal dominant inheritance. The exact incidence of distal arthrogryposis is not known.[5]

c. Arthrogryposis may also be present in different syndromes and diseases (ie, Larsen syndrome, myopathy, diastrophic dysplasia, pseudotrisomy $X$, myopathy, trisomy 18). The incidence 
of these disorders is collectively estimated to be between 1 in 3000 to 5100 live births.[5] Intellectual disability and consanguinity are often seen in this group of disorders. [1]

Although the different categories of arthrogryposis have different etiopathogenesis, all forms are associated with decreased fetal movement (fetal akinesia).[5] The latter may be the result of abnormalities of muscle, nerve, connective tissue, space limitation within the uterus, maternal disorders and maternal exposure to medications, drugs and environmental factors $[5,6]$. These above-mentioned causative factors are postulated to produce secondary contractures.[3]

We present a rare case of lower limb amyoplasia presenting with severe scoliosis and multiple skin dimples in an unusual location (bilateral gluteal area). The study was performed according to the CARE statement.

\section{CASE PRESENTATION}

This is a case of an 11 -year-old female, born of a non-consanguineous marriage, who presented with severe scoliosis. The study was performed according to the CARE guidelines for the case report.

The patient was born to a 22-year-old gravida 1 para 1 (GIP1) mother whose pregnancy was not documented until the fourth month age of gestation
(AOG). The pregnancy was an incidental finding during a consultation for urinary tract infection. The mother mentioned she did not expect to be pregnant as her abdomen was not enlarged compared to usual. At about this time, the mother experienced crampy hypogastric pain and bleeding episodes. She was seen by an obstetrician-gynecologist at a local hospital where she was diagnosed to have threatened abortion and was advised bed rest with a prescription of unrecalled luteal support medications. This was resolved and succeeding prenatal checkups and ultrasound reportedly revealed normal fetal heartbeat and movement. The mother, however, described fetal movement to be few and weak in intensity, even until the $36^{\text {th }}$ week AOG. The mother had no known exposures to rubella, irradiation, or noxious agents. The family history was noncontributory.

The patient was delivered term at 38 weeks AOG with a birth weight of $2.8 \mathrm{kgs}$ via caesarian section due to a breech presentation, with APGAR scores of 3 and 9 at the first and fifth minute of life, respectively.

According to the mother, the pediatrician informed her that the umbilical cord was wrapped around the infant's neck upon delivery, and that there were findings of occult blood in the stool. The mother also reported the following findings of the pediatrician regarding the patient: (1) a prominent forehead described to be longer than wide in shape, (2) fingers observed to be in a slightly clenched position with
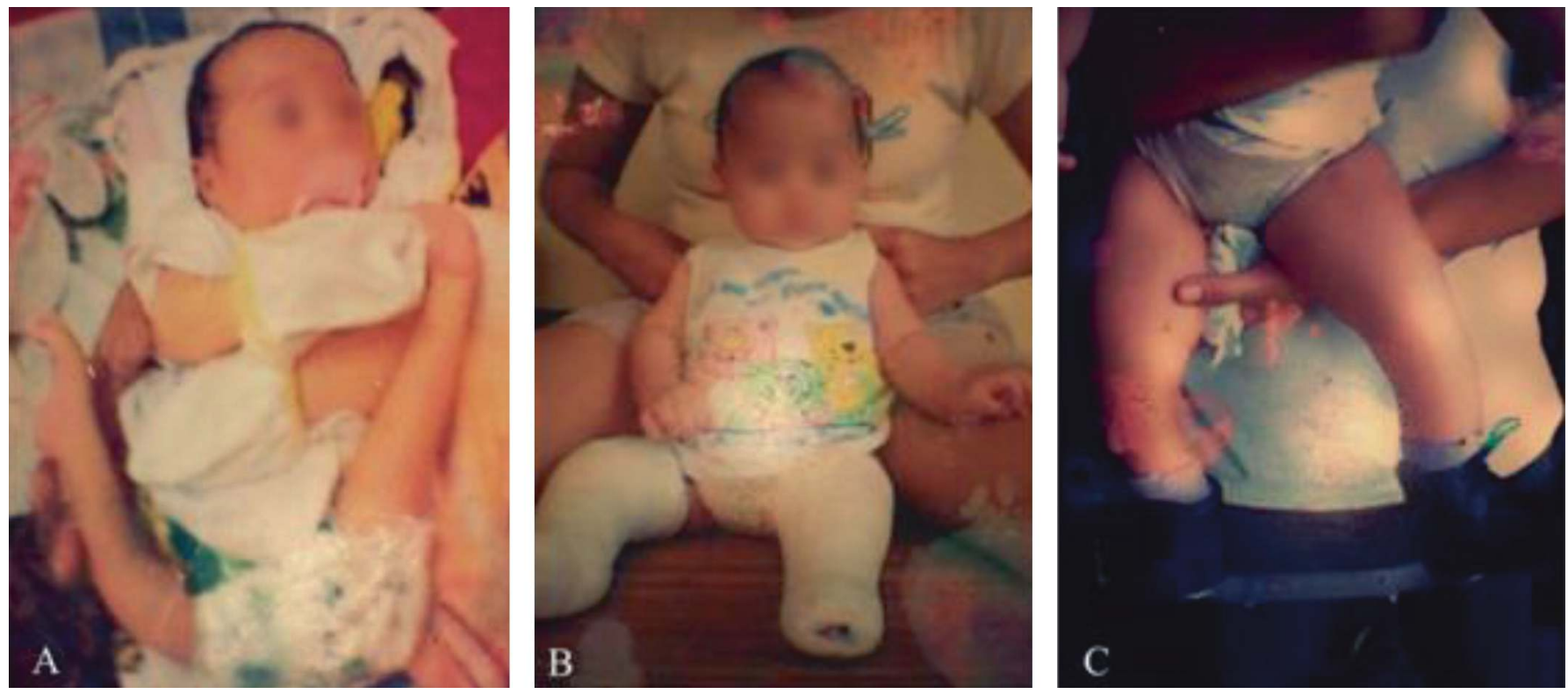

Figure I. The patient's appearance at (A) birth, (B) 6 months, and (C) 9 months. A. Hips are flexed and the legs are externally rotated with bilateral genu recurvatum deformity. B. Patient is unable to sit independently. Flexion-adduction deformity of the thumb.

C. Patient in ankle-foot orthosis. Bilateral patella is not prominent. 

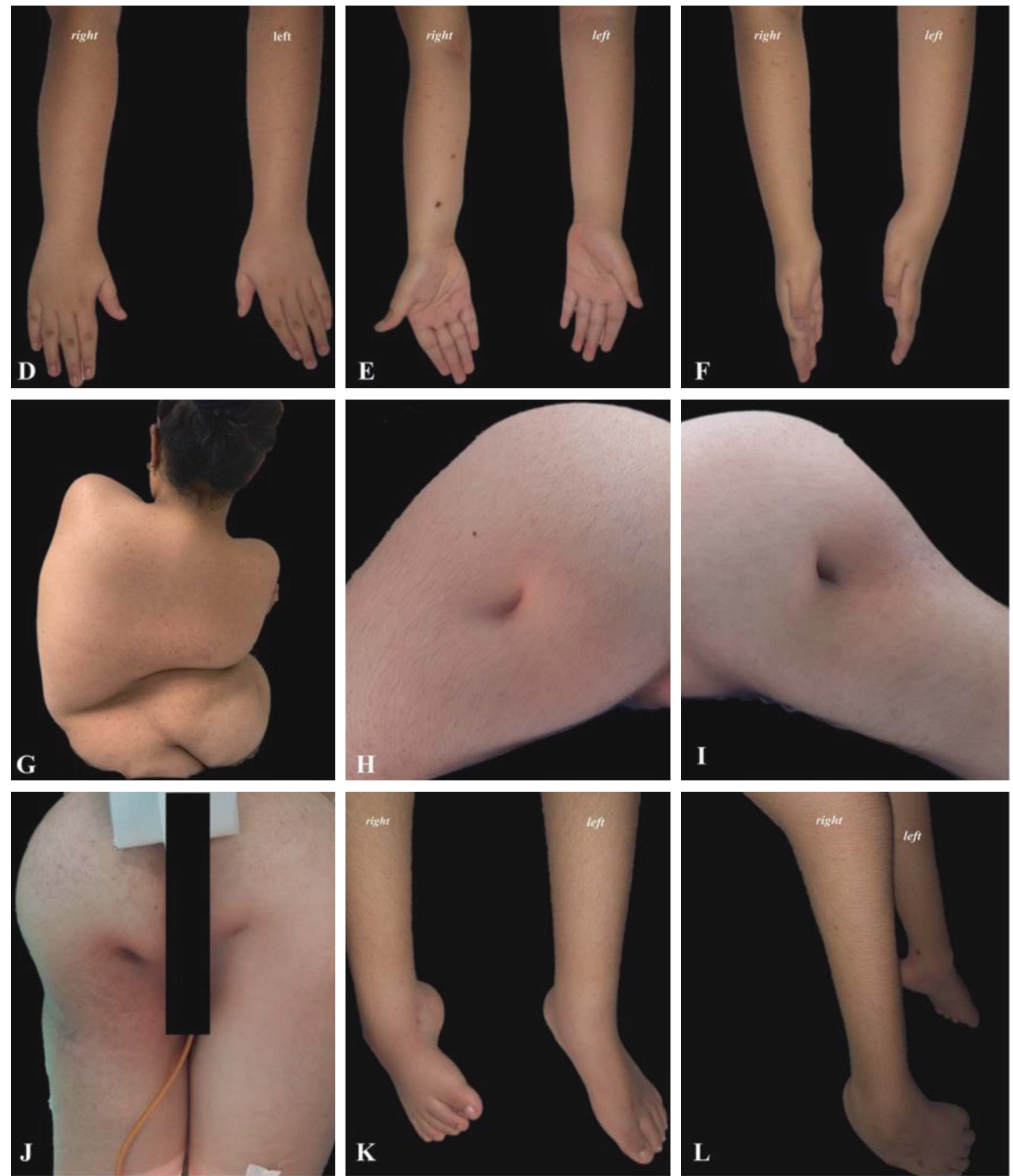

Figure II. Patient's physical examination findings. D. The distal interphalangeal creases are not appreciated in the left hand. Ulnar deviation of the left wrist. E. Shortened left limb. F. Flexion contracture of the interphalangeal joints on the left hand. G. Severe lateral curvature of the spine with pelvic obliquity. H. Dimpling over the right greater trochanteric area. I. Dimpling over the left greater trochanteric area. J. Bilateral dimpling over the ischial tuberosity. Flattening of buttocks. K. Right foot clubbed in equinovarus. Dimpling over the medial aspect of the ankle. L. Atrophy of the lower extremities. 


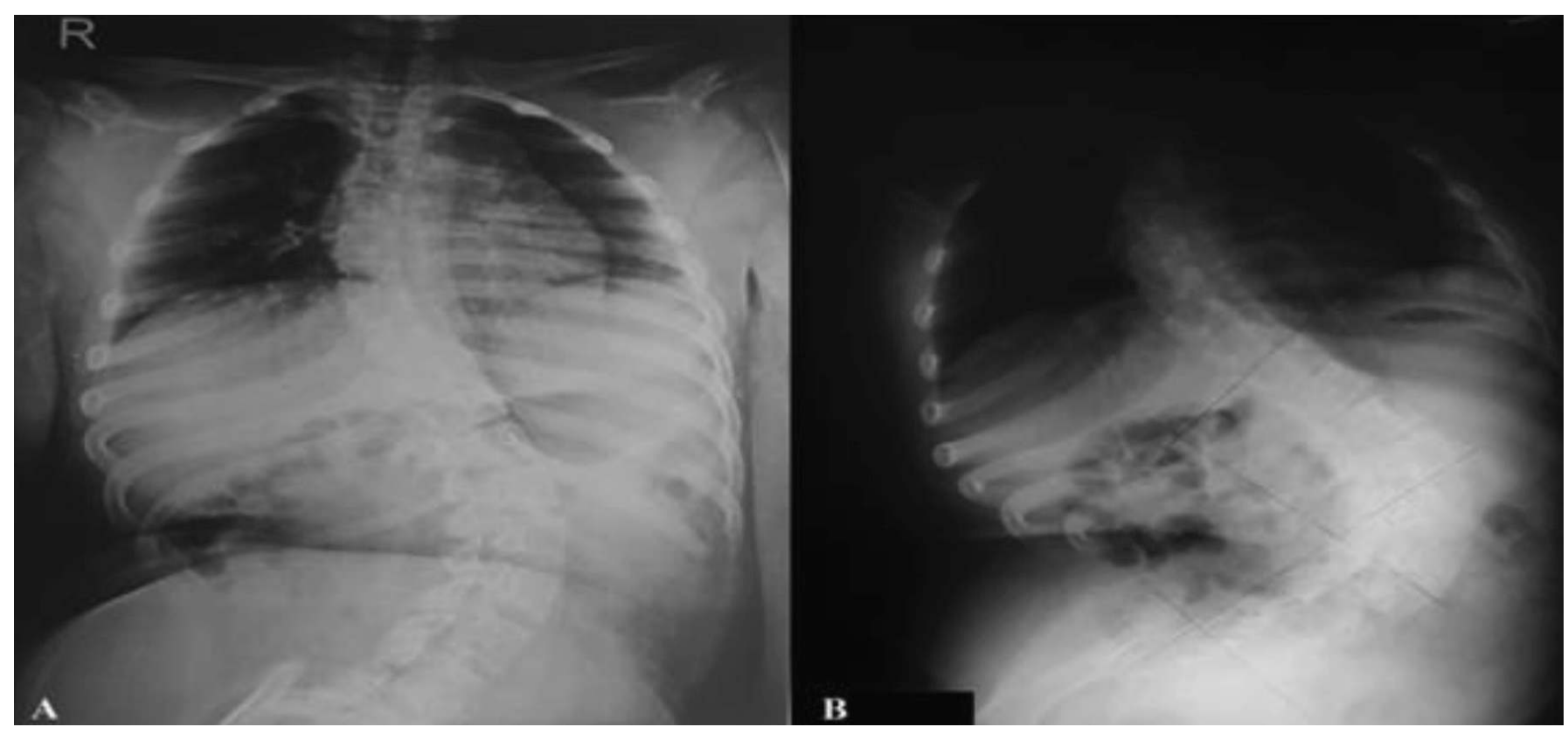

Figure III. Thoracolumbar spine. Anteroposterior view. A. Cobb's angle, thoracic 40 degrees and lumbar 60 degrees (July 2017 ). B. Cobb's angle, thoracic level 45 degrees and lumbar level 79.5 degrees (May 2018).

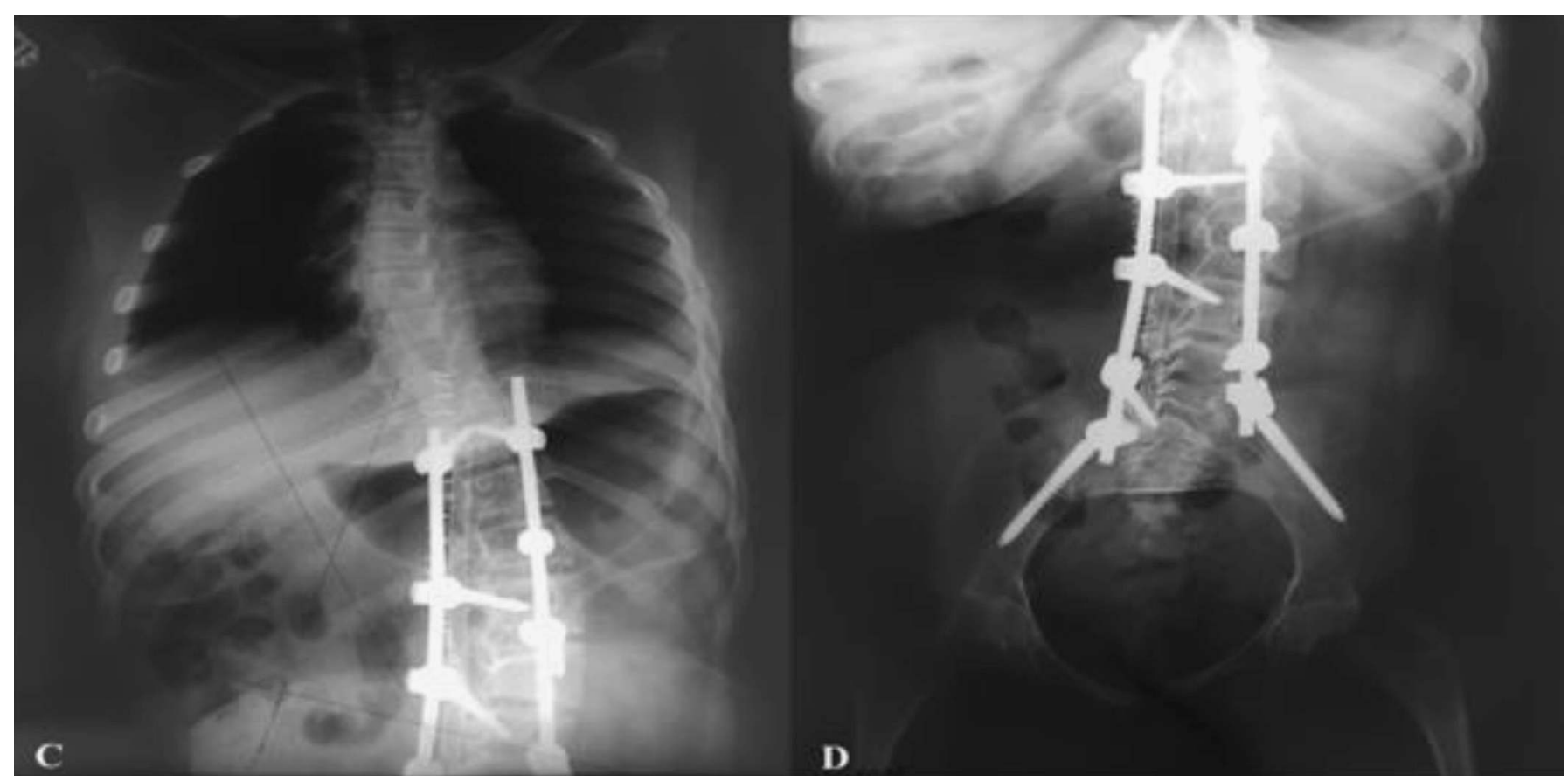

Figure IV. Scoliosis corrective surgery. Posterior instrumentation from T10 to L5 with pelvic fixation using iliac screws. Cobb's angle, thoracic level 25 degrees and lumbar level Cobb's angle 42 degrees.

the thumb drawn into the palm, (3) hips flexed with knee hyperextension such that the feet remained close to the face, (4) multiple, bilateral and symmetrical skin dimples over the gluteal area, (5) very little muscle tissue with weak muscle tone on both legs, (6) bilateral patella not appreciated, (7) symmetrical contractures on both lower extremities, (8) and clubbing of both feet (Figure IA). The rest of the physical examination was otherwise unremarkable.
The patient was presumptively treated for asphyxia neonatorum and necrotizing enterocolitis. She was discharged stable on the $5^{\text {th }}$ hospital day with a final diagnosis of arthrogryposis.

At 1 month of age, the patient underwent physical therapy consisting of gentle manipulation and stretching of the hyperextended knees opposite the direction of the deformity at a local hospital. At 6 months of age, the patient underwent serial bilater- 


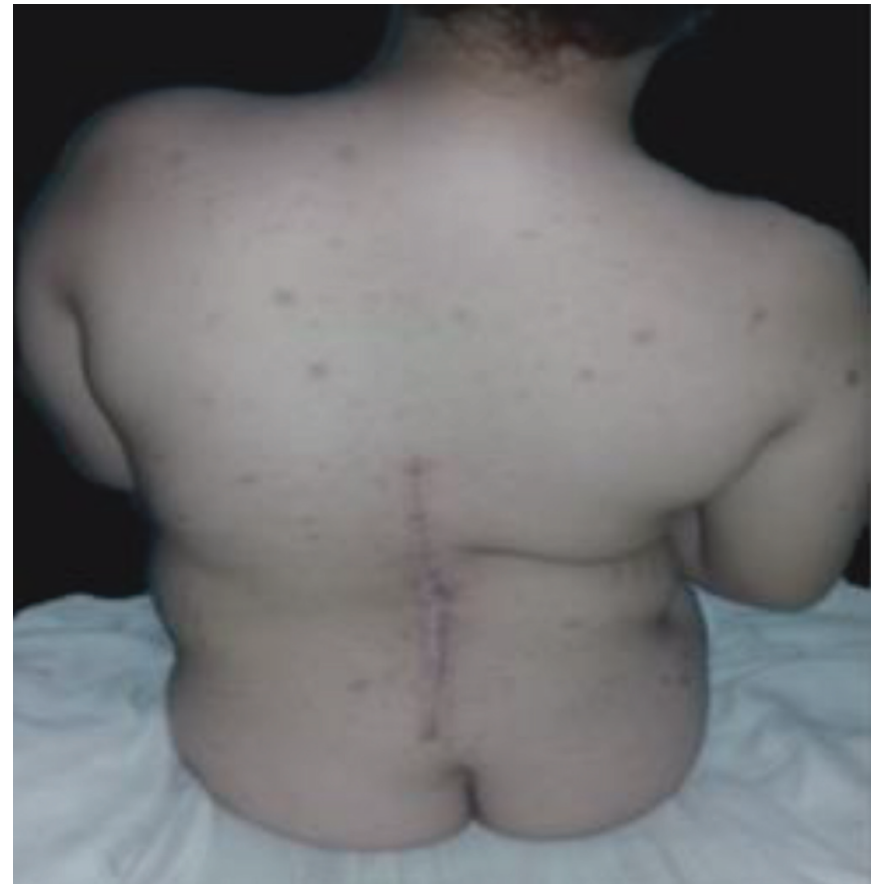

Figure V. Third month post-surgery. More stable sitting posture with both gluteal areas receiving equal weight.

al Ponseti casting with percutaneous release of the Achilles tendon (Figure IB). Foot abduction braces were used indefinitely post-surgery. At 3 years of age, the mother noted recurrence of the clubfoot deformity as well as curvature of the spine. The patient was then referred to a pediatric neurologist wherein an MRI of the spine was requested, reportedly showing normal results. No other diagnostic procedures were requested.

Interval history revealed a rapid progression of the spinal curvature.

At 9 years of age, the mother observed prolonged cough with further worsening of the spinal curvature (Figure III). The increasing severity of scoliosis prompted referral to a spine surgeon in our institution.

Upon examination, the patient was conscious, coherent, and not in distress. The patient was non-ambulatory and wheel-chair-borne. The head, eyes, ears, nose, throat, heart, and lung findings were unremarkable. The cranial nerves were intact. There were no sensory deficits observed in both the upper and lower extremities. There is ulnar deviation of the left wrist. Distal interphalangeal creases were not appreciated in the left hand (Figure IID). There is full range of motion at the elbow, wrist, and digits of both upper extremities. Manual muscle testing was $5 / 5$ in both upper extremities. The spine has a severe leftward curvature with pelvic obliquity (Figure II G). There are two symmetrical areas of skin dim- pling observed over the greater trochanteric area (Figure $\mathrm{II} \mathrm{H}$ ) and the ischial tuberosity area (Figure II I). The patient is able to actively extend both hips up to about 0-10 degrees. The lower extremities are grossly atrophied with talipes equinovarus of the right foot, and there is smoothening of the creases overlying the knee (Figure II K-L). The patient is unable to do active motion on the lower extremities.

The patient's personal, social, and verbal skills were found to be on par with her age. She is currently in fourth grade and has been reported to be performing well academically. Motor deficits are in line with her current condition.

Based on the symmetrical contractures observed in the lower extremities at birth, the patient was diagnosed to have arthrogryposis. Corrective spine surgery was advised to correct scoliosis.

The sitting balance was a major concern for the patient because of the pelvic tilt (Figure II G). In order to gain pelvic control and assume a balanced pelvis, we utilized one iliac screw on each side of the pelvis (Figure IV C, D). This resulted in a more stable sitting posture with both gluteal areas receiving equal weight (Figure V). Even though the patient presented with a double major curve with a Cobb's angle of 49.7 and 79.5 degrees of the thoracic and lumbar curves, respectively (Figure III B), the surgeon decided to end the construct at T10 to allow some residual flexibility to the upper spine in order to preserve the patient's capability of propping herself to sit from a recumbent position. Further follow-up will be necessary to observe the compensatory curve's development.

Figure IV C, D shows the x-ray of the patient three months after surgery with Cobb's angle of 25 degrees and 42 degrees thoracic and lumbar curves, respectively.

Three months after spine correction surgery, the patient is now able to sit without support. The main goal of management is to improve her independence. Future plans include child counseling and pediatric physiatrists referral for upper limb strengthening and wheelchair transfers.

\section{DISCUSSION}

The diagnosis of arthrogryposis in our patient was made due to the presence of multiple congenital contractures. The salient features of lower limb contractures, normal intellect, absence of a family history 
of congenital contractures, and no other organ malformations suggest a diagnosis of amyoplasia, the sporadic, classical type of arthrogryposis, which presents with reduced muscle mass, multiple joint contractures, characteristic fixed limb positions, and normal intellect.[1]

Amyloplasia $(A=n o, m y o=m u s c l e$, plasia $=$ growth $)$ has an estimated incidence of 1 in 10,000 live births. [3] Based on a study by Hall, et al, amyoplasia may be divided into five disease subtypes based on the affected extremity: (1) Symmetric upper and lower extremity involvement $(55.9 \%)$, (2) Severe symmetric upper and lower extremity involvement $(7.3 \%),(3)$ Three limb involvement $(4.8 \%),(4)$ Upper limb involvement alone (16.8\%), and (5) Lower limb involvement alone (15.5\%).[3] Despite some affectation of her upper extremity, the patient's predominant presentation was the symmetrical congenital contractures of both lower extremities, which supports a diagnosis of lower limb amyoplasia.[3]

The incidence of lower limb-only amyoplasia is estimated to be 1 in 75,000 to 1 in 100,000. Only 85 cases have been identified in literature, and there are no published cases from the Philippines (Pubmed, Cochrane, EMBASE, grey literature, ie, review of medical records, an inquiry from other training institutions).[7]

Aside from the rare occurrence of lower limb amyoplasia, our patient also presented with two uncommon features within this patient group: severe scoliosis and large, deep skin dimpling located in an unusual area.

Scoliosis is not frequently seen in amyoplasia. In a cohort of 560 patients with amyoplasia (all types), only $63(11 \%)$ had scoliosis. Out of the 560, 85 had lower limb amyoplasia and only 6 of these presented with concomitant scoliosis. [8] Similar to our patient's case, scoliosis seen in these patients was rigid, rapidly progressive, and often not present at birth. $[8,11]$ The most common spinal deformity present in these patients was a single long thoracic curve. $[8,11]$ In contrast, our case presented with a rigid, rapidly progressive double thoracolumbar curve scoliosis with pelvic obliquity. This has only been reported once in literature.[11] In addition, these cases with concomitant scoliosis were often of the mild type (Cobb's angle $<40$ degrees), while our case had a severe type of scoliosis.

Aside from severe scoliosis, another unique feature of the patient was the presence of large, deep skin dimpling in an unusual area. Skin dimpling overlying the affected joint with contractures is often observed in patients with amyoplasia.[3] However, dimpling is neither very deep nor very large and does not usually occur in the buttocks, sacrum, chest, sternum, and inner arm.[9] The occurrence of deep skin dimples in these unusual locations has been documented in thirty cases of arthrogryposis associated with oligohydramnios. Only one of the cases in this cohort had multiple dimples in the buttocks area.[9] Our patient manifested with multiple bilateral gluteal dimpling, which seems to be unusual in amyoplasia. Unfortunately, the amniotic fluid was not quantitatively measured to ascertain causation in this case. However, prenatal history and clinical signs reported by the mother (decrease in perceived fetal movement) may suggest oligohydramnios in this case.

The prognosis of arthrogryposis is dependent on the underlying pathology. Conditions with multi-organ association may cause early mortality, while those with less severe affectation have a normal life expectancy, yet may require extensive orthopedic treatment follow-up and rehabilitation. [4] The latter is the likely case for our patient as she has no other deficits other than her joint contractures and scoliosis. Although amyoplasia does not significantly alter life expectancy, it is a lifelong disorder that can severely affect the quality of life.[3] In general and as is the case of our patient, surgery may be indicated for the correction of joint contractures. [4] As severe scoliosis is not usually present in amyoplasia, there are no available studies on the prognosis and outcome of severe scoliosis in these patients treated with corrective surgery.[8]

Unfortunately, $75 \%$ of arthrogryposis cases are not diagnosed prior to delivery because the fetal movement is not routinely assessed sonographically. It is quite challenging to detect fetal movement of each limb area because this requires technical expertise and time [10], which is not often available, especially in rural settings. It is thought that if fetal akinesia is detected early, fetal movement may be increased using methods such as maternal exercise, potentially leading to less severe contractures.[5] In the case of our patient, maternal concern about the lack of fetal movement could have been addressed further through more detailed ultrasound studies, which in turn could lead to methods to increase fetal movement, alleviating the condition to some extent. 


\section{CONCLUSION}

We presented a rare case of a Filipino child with lower limb-only amyoplasia with additional unique features of severe scoliosis and multiple, large and deep dimples on the gluteal area. This case report further highlights not only the importance of antenatal surveillance but attention to the reported signs and symptoms.

\section{Ethical Considerations}

The information that we have collected which may lead to the identification of the patient such as the name, date of birth, medical record number, geographic location, and phone numbers were not disclosed to comply with the principles of anonymity and confidentiality. Both the written and verbal consent were given by the parents of the patient. The parents voluntarily agreed to provide the information for this case, which included the gross photographs of the patient and radiographic images which were used for the purpose of research.

\section{CONFLICT OF INTEREST}

I have no affiliations with or involvement in any organization or entity with any financial interest, or non-financial interest in the subject matter discussed in this manuscript. 


\section{REFERENCES}

1. Hall JG, Reed SD, Driscoll EP, Opitz JM. Part I. Amyoplasia: a common, sporadic condition with congenital contractures. American Journal of Medical Genetics. 1983 Aug; 15(4):571-90.

2. Hall JG. Arthrogryposis (multiple congenital contractures): diagnostic approach to etiology, classification, genetics, and general principles. European Journal of Medical Genetics. 2014 Aug 1;57(8):464-72.

3. Hall JG, Aldinger KA, Tanaka KI. Amyoplasia revisited. American Journal of Medical Genetics Part A. 2014 Mar; 164(3):700-30.

4. Bevan WP, Hall JG, Bamshad M, Staheli LT, Jaffe KM, Song K. Arthrogryposis multiplex congenita (amyoplasia): an orthopaedic perspective. Journal of Pediatric Orthopaedics. 2007 Jul 1;27(5):594-600.

5. Hall JG. Arthrogryposis (multiple congenital contractures): diagnostic approach to etiology, classification, genetics, and general principles. European Journal of Medical Genetics. 2014 Aug 1;57(8):464-72.

6. Swinyard CA. Concepts of multiple congenital contractures (arthrogryposis) in man and animals. Teratology. 1982 Apr;25(2):247-58.

7. Hall JG. Amyoplasia involving only the upper limbs or only involving the lower limbs with review of the relevant differential diagnoses. American Journal of Medical Genetics Part A. 2014 Apr; 164(4):859-73.

8. Agranovich OE, Komolkin IA. Scoliosis in patients with arthrogryposis: a literature review. Pediatric Traumatology, Orthopaedics and Reconstructive Surgery. 2017 Oct 9;5(3):93-8
9. Hall JG. Oligohydramnios sequence revisited in relationship to arthrogryposis, with distinctive skin changes. American Journal of Medical Genetics Part A. 2014 Nov; 164(1 1):2775-92.

10. Filges I, Hall JG. Failure to identify antenatal multiple congenital contractures and fetal akinesia-proposal of guidelines to improve diagnosis. Prenatal Diagnosis. 2013 Jan;33(1):61-74.

11. Yingsakmongkol W, Kumar SJ. Scoliosis in arthrogryposis multiplex congenita: results after nonsurgical and surgical treatment. Journal of Pediatric Orthopaedics. 2000 Sep $1 ; 20(5): 656-61$.

cc) Open Access This article is licensed under a Creative Commons Attribution 4.0 International License, which permits use, sharing, adaptation, distribution and reproduction in any medium or format, as long as you give appropriate credit to the original author(s) and the source, provide a link to the Creative Commons license, and indicate if changes were made. The images or other third party material in this article are included in the article's Creative Commons license, unless indicated otherwise in a credit line to the material. If material is not included in the article's Creative Commons license and your intended use is not permitted by statutory regulation or exceeds the permitted use, you will need to obtain permission directly from the copyright holder. To view a copy of this license, visit http://creativecommons.org/licenses/ by/4.0/. 